\title{
Multi-objective trajectory optimization of Space Manoeuvre Vehicle using adaptive differential evolution and modified game theory
}

\author{
Runqi Chai ${ }^{\mathrm{a}}$, Al Savvaris ${ }^{\mathrm{a}}$, Antonios Tsourdos ${ }^{\mathrm{a}}$, Senchun Chai ${ }^{\mathrm{b}, *}$ \\ ${ }^{a}$ School of Aerospace, Transport and Manufacturing, Cranfield University, Bedfordshire, \\ MK43 OAL, United Kingdom \\ ${ }^{b}$ School of Automation, Beijing Institute of Technology, Beijing 100081, PR China
}

\begin{abstract}
Highly constrained trajectory optimization for Space Manoeuvre Vehicles (S$\mathrm{MV}$ ) is a challenging problem. In practice, this problem becomes more difficult when multiple mission requirements are taken into account. Because of the nonlinearity in the dynamic model and even the objectives, it is usually hard for designers to generate a compromised trajectory without violating strict path and box constraints. In this paper, a new multi-objective SMV optimal control model is formulated and parameterized using combined shooting-pseudospectral technique. A modified game theory approach, coupled with an adaptive differential evolution algorithm, is designed in order to generate the pareto front of the multi-objective trajectory optimization problem. In addition, to improve the quality of obtained solutions, a control logic is embedded in the framework of the proposed approach. Several existing multi-objective evolutionary algorithms are studied and compared with the proposed method. Simulation results indicate that without driving the solution out of the feasible region, the proposed method can perform better in terms of convergence ability and convergence speed than its counterparts. Moreover, the quality of the pareto set generated using the proposed method is higher than other multi-objective evolutionary algorithms, which means the newly proposed algorithm is more attractive for solving multi-criteria SMV trajectory planning problem.
\end{abstract}

\footnotetext{
${ }^{*}$ Corresponding author

Email addresses: r.chai@cranfield.ac.uk (Runqi Chai), a.savvaris@cranfield.ac.uk (Al Savvaris), a.tsourdos@cranfield.ac.uk (Antonios Tsourdos), chaisc97@bit.edu.cn (Senchun Chai)
}

Preprint submitted to Journal of ${ }^{A} T_{E} X$ Templates

April 3, 2017

Published by Elsevier. This is the Author Accepted Manuscript issued with: Creative Commons Attribution Non-Commercial No Derivatives License (CC:BY:NC:ND 4.0). The final published version is available online at 10.1016/j.actaastro.2017.02.023. Please refer to any applicable publisher terms of use. 
Keywords: trajectory optimization, Space Manoeuvre Vehicles, modified game theory, adaptive differential evolution, multi-objective evolutionary algorithms.

\section{Introduction}

In the future, it is very likely that Space Manoeuvre Vehicles (SMV) are going to play an increasingly important role in space exploration. Therefore, a well-planned trajectory, particularly in skip entry phase, is key to stable flight 5 and improved guidance control of the vehicle [1-6]. Unlike studies investigated in most of literatures [1, 7], where the re-entry problem was addressed, the mission scenario studied in this paper focuses on the atmospheric skip hopping, targeting the entry into the atmosphere down to a predetermined position (predetermined altitude was set at the start of this project to specifically address this type of mission) and the required controls involved in returning back to low Earth orbit. Commonly, these types of trajectory planning problem can be described as an optimal control problem and numerical methods are usually applied to calculate the near-optimal solution [8].

Numerical methods for solving trajectory optimization problems are divided into two major classes: indirect methods and direct methods [8 13. However, it is difficult to solve the trajectory design problem using indirect methods, since the maximum principle is required to be derived. Therefore, direct optimization method has been widely used for trajectory optimization. Among direct methods, one traditional technique which has been used in practical problems

20 is direct multiple shooting method [1, 4, 14. The main process of the direct multiple shooting method is to parameterize only the control variables using interpolation at discretized time nodes. In recent years, collocation methods for discretizing optimal control problems have increased in popularity. Unlike shooting methods, collocation techniques parameterize both the state and con25 trol variables. There are two kinds of collocation methods, local collocation method (e.g. the direct collocation) and global collocation method (e.g. the pseudospectral method [15]). Two well-known pseudospectral methods for solving trajectory optimization problems are the Legendre pseudospectral method [11, 12, 16, 17] and the Chebyshev pseudospectral method [18, 19]. Compared 30 with multiple shooting method, collocation methods tend to result in large scale optimization parameters and it has not been used in real application to date. A 
main reason is that current onboard computational devices may fail to satisfy the requirement of the large computational time needed for these algorithms. Therefore, a hybrid multiple shooting scheme is proposed in this paper. This

35 method can keep the advantages of using collocation scheme but not result a large scale nonlinear programming problem so that the computational burden of the optimizer can be reduced.

In most of existing studies, the trajectory planning problem usually aim$\mathrm{s}$ at one single objective, for example, minimizing the aerodynamic heating, 40 maximizing the final velocity, etc. However, most of the real-world design problems encountered by aerospace engineers involve simultaneous optimization of several competitive objective functions 2022]. For the mission considered in this paper, the expectations for enhancing performance and saving cost are of significant importance. Therefore, it is desired to have a multi-objective SMV

45 model with multiple criteria so as to capture more of the real-world requirements. There are many multi-objective methods, which are suitable for these kind of problems. Since the solution of multi-objective programming problem is not unique (known as nondominated solution), multi-objective evolutionary algorithms are commonly implemented to generate all the potential solutions 50 (also known as pareto set) 23 28]. Deb et al. 23] developed the Nondominated Sorting Genetic Algorithm (NSGA2) using a nondominated sorting procedure and the crowding distance metric, and in [29], NSGA2 method was applied to generate the pareto front of a multi-objective re-entry trajectory optimization problem. Li et al.21] applied a Multi-objective Evolutionary Algorithm Based 55 on Decomposition (MOEA/D) method to solve general multi-objective problems. However, from the previous works, it was analyzed that the computational burden of iterative optimization is heavy and the quality of obtained solution still needs to be improved. Hence, in this paper, an adaptive differential evolution based on modified game theory algorithm is designed. By applying the so control logic, the quality of the generated solution can be improved. Moreover, in order to enhance the convergence ability, a modified game theory is introduced and coupled in the algorithm framework.

It is worth noting that one practical use of the obtained theoretic results is that it can be applied to design the online guidance law or used as the reference 65 command for the online tracking algorithm [5, 7]. The main advantage for tracking these reference trajectories is that all the reference results can satisfy 
the constraints and multiple mission-dependent objectives can be optimized. Since the pareto front information can be calculated by applying the proposed Multi-objective trajectory optimization method, the decision makers can have more flexibility to do the decision-making based on the presented relationships between different conflicting objective functions.

Hereafter, the paper is organised as follows. In Section 2, a new 3-DOF continuous-time optimal control model including different types of constraints and objective functions is established and parameterized using the hybrid multiple shooting method. Following that, Section 3 presents the framework of proposed adaptive differential evolution and modified game theory algorithm. Compromised solutions generated by employing the proposed method and different evolutionary multi-objective approaches are given in Section 4. The paper ends with Section 5, the conclusions.

\section{Problem formulation}

The skip entry can be divided into five phases: initial roll, down control, up control, Kepler and final entry. Considering the mission of the SMV is to overfly the ground target with specific altitude, the most challenging down control and up control will be considered in this paper. The overall mission is illustrated in Fig.1.

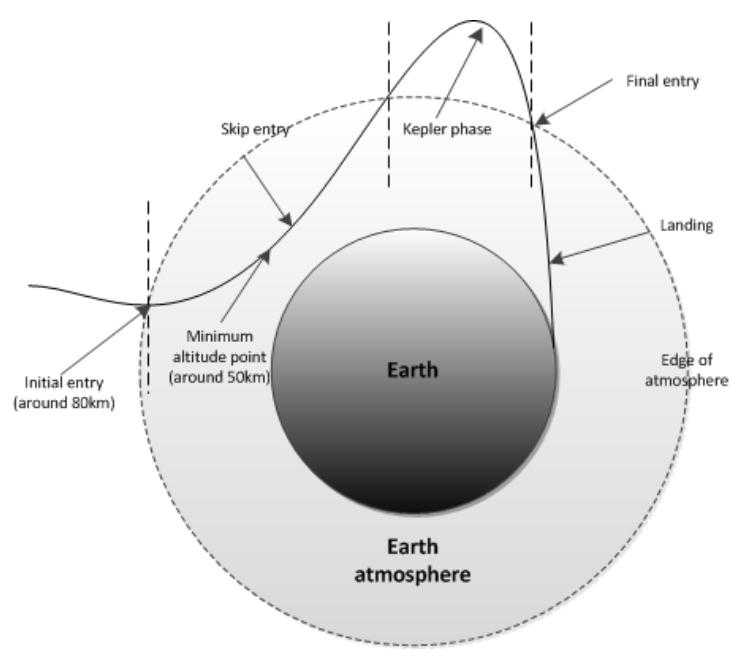

Figure 1: General mission profile 


\subsection{Dynamic model}

Taking the rotation of the Earth into account, the 3-DOF equations of motion of the space manoeuvre vehicle are constructed by the following set of Ordinary Differential Equations (ODEs):

$$
\begin{gathered}
\dot{r}=V \sin \gamma \\
\dot{\theta}=\frac{V \cos \gamma \sin \psi}{r \cos \phi} \\
\dot{\phi}=\frac{V \cos \gamma}{r} \psi \\
\dot{V}=\frac{T \cos \alpha-D}{m}-g \sin \gamma+\Omega^{2} r \cos \phi(\sin \gamma \cos \phi-\cos \gamma \sin \phi \cos \psi) \\
\dot{\gamma}=\frac{L \cos \sigma+T \sin \alpha}{m V}+\left(\frac{V^{2}-g r}{r V}\right) \cos \gamma+2 \Omega \cos \phi \sin \psi \\
+\Omega^{2} r \cos \phi(\cos \gamma \cos \phi+\sin \gamma \cos \psi \sin \phi) V^{-1} \\
\dot{\psi}=\frac{L \sin \sigma}{m V \cos \gamma}+\frac{V}{r} \cos \gamma \sin \psi \tan \phi+\frac{\Omega^{2} r \sin \psi \cos \phi \sin \phi}{V \cos \gamma} \\
-2 \Omega(\tan \gamma \cos \psi \cos \phi-\sin \phi) \\
\dot{m}=-\frac{T}{I_{s p} g}
\end{gathered}
$$

$$
\begin{array}{ll}
g=\frac{\mu}{r^{2}} & \rho=\rho_{0} \exp \frac{r-r_{e 0}}{h_{s}} \\
L=\frac{1}{2} \rho V^{2} C_{L} S & D=\frac{1}{2} \rho V^{2} C_{D} S \\
C_{D}=C_{D 0}+C_{D 1} \alpha+C_{D 2} \alpha^{2} & C_{L}=C_{L 0}+C_{L 1} \alpha
\end{array}
$$

where $S=249.91 \mathrm{~m}^{2}$ is reference area, $\rho$ is the density of the atmosphere and $\rho_{0}=1.2250 \mathrm{~kg} / \mathrm{m}^{3}$ is the density of the atmosphere at sea-level. $r_{e 0}=$ $6378.135 \mathrm{~km}$ is earth radius, $L$ and $D$ are the lift and drag whereas $C_{L}$ and $C_{D}$ are the corresponding lift and drag coefficients. $g$ is the gravitational acceleration. Although $g$ can be treated as a constant, to make the problem more realistic, it is assumed that the gravitational acceleration is varying with respect to the altitude.

A detailed description in terms of the entry reference frames and aerodynamic forces can be found in Fig.2.

In the model given by Eq.(1), three autopilot equations are introduced by using the technique of first order lag [30] to describe the rate constraint of the 


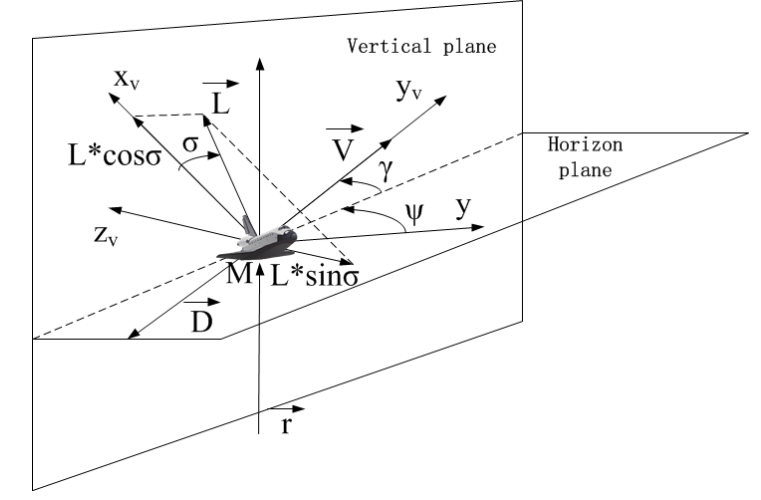

Figure 2: Entry vehicle and aerodynamic forces

controls,

$$
\begin{aligned}
\dot{\alpha} & =K_{\alpha}\left(\alpha_{c}-\alpha\right) \\
\dot{\sigma} & =K_{\sigma}\left(\sigma_{c}-\sigma\right) \\
\dot{T} & =K_{T}\left(T_{c}-T\right)
\end{aligned}
$$

where $\alpha_{c}, \sigma_{c}$ and $T_{c}$ are the demanded angle of attack, bank angle and thrust, respectively. Correspondingly, three new control constraints should be introduced.

$$
\begin{aligned}
\alpha_{c(\min )} & \leq \alpha_{c} \leq \alpha_{c(\max )} \\
\sigma_{c(\min )} & \leq \sigma_{c} \leq \sigma_{c(\max )} \\
T_{c(\min )} & \leq T_{c} \leq T_{c(\max )}
\end{aligned}
$$

The box constraints and aerodynamic parameters are applied using the same definitions in 20], whereas the path constraints are aerodynamic heating, dynamic pressure and load factor.

$$
\begin{gathered}
\dot{Q}_{d}=K_{Q} \rho^{0.5} V^{3.07}\left(c_{0}+c_{1} \alpha+c_{2} \alpha^{2}+c_{3} \alpha^{3}\right)<\dot{Q}_{d \max } \\
P_{d}=\frac{1}{2} \rho V^{2}<P_{d \max } \\
n_{L}=\frac{\sqrt{L^{2}+D^{2}}}{m g}<n_{\operatorname{Lmax}}
\end{gathered}
$$

where $Q_{d \max }, P_{d \max }$ and $n_{L \max }$ represents acceptable maximum heating rate, dynamic pressure and acceleration, respectively.

\subsection{Objective functions}

In the past, early studies on spacecraft trajectory optimization problems usually focussed on single objective. However, in order to take more practical 
requirements into account, this type of problem should be constructed containing multiple objectives [28, 31]. Nowadays the majority of research is focusing on that. In this paper four objectives are considered to capture more realities of the problem. The first objective function to be minimized is the final time such that the reconnaissance mission is completed in the shortest possible time interval. In addition, as mentioned in [20, minimizing the total aerodynamic heating is also chosen as one of the objectives since the vehicle structure integrity is largely affected by the aerodynamic heating. The third objective is to maximize the final velocity to increase the kinetic energy and, therefore, raise the flexibility to maneuver the vehicle back into the orbit. Moreover, in this mission scenario to ensure the SMV has enough fuel to carry-out several skip hops, the final objective is set to minimize the fuel consumption, i.e., maximize the final mass value, during the whole manoeuvre. Therefore, the objective functions selected for the analysis are:

1). Minimizing the final time:

$$
\min J_{1}=t_{f}
$$

2). Minimizing the total aerodynamic heating:

$$
\min J_{2}=\int_{t_{0}}^{t_{f}} \dot{Q}(t) d t
$$

3). Maximizing the final velocity:

$$
\max J_{3}=V\left(t_{f}\right)
$$

4). Maximizing the final mass:

$$
\max J_{4}=m\left(t_{f}\right)
$$

\subsection{Discrete method}

To solve the continuous SMV trajectory hopping problem, discrete algorithms should be implemented so that the continuous optimal control problem can be converted to static nonlinear programming (NLP) problem. The discrete method used in this paper combines multiple shooting and pseudospectral method together. The control variables can be approximated by interpolation at $N_{k}$ th discretized time nodes $\left[\tau_{1}, \tau_{2}, \ldots, \tau_{N_{k}}\right]$. Then the equations of motion are integrated with a fourth order Runge-Kutta method. In order to improve 
the approximation of control sequence, the orthogonal interpolation is applied, which means the control is approximated as:

$$
u(\tau) \approx U(\tau)=\sum_{i=0}^{N} U_{i} L_{i}(\tau)
$$

where $\tau \in[-1,1], L_{i}(\tau),(i=0, \ldots, N)$ are the collocation points and a basis of Lagrange polynomials, respectively. The Legendre-Gauss-Radau (LGR) points are used as the node points. LGR points are the root of linear combination of

Legendre polynomials which can be written as:

$$
P_{K-1}(\tau)+P_{K}(\tau)=0
$$

where the $K t h$ order Legendre polynomial $P_{K}(\tau)$ is

$$
P_{K}(\tau)=\frac{1}{2^{K} K !} \frac{d^{K}}{d \tau^{K}}\left[\left(\tau^{2}-1\right)^{K}\right]
$$

By using the approach described above, the SMV trajectory optimization problem is converted to NLP with control variables at collocation nodes.

\section{Adaptive differential evolution based on modified game theory}

The proposed method is based on the genetic class of evolutionary algorithms (EA), which generates solutions to optimization problems taking inspiration from natural selection and survival of the fittest in the biological world. This algorithm can be used as a distributed behavioural algorithm which can perform a multi-dimensional search to find the solution of various optimization problems. There are four main procedures for the proposed algorithm, initialization, selection, crossover and mutation. In the initialization part, after the scale of the population and the number of iterations are assigned, the first population needs to be generated. According to the discretization method described in Eq.10 to Eq.12, the optimization parameters (demanded angle of attack $\alpha_{c}$, bank angle $\sigma_{c}$ and thrust $T_{c}$ ) should be initialized at all the discrete time nodes, which can satisfy the control constraints described in Eq.(4). This can be written as:

$$
U_{i j, G}=U_{l_{j}}+\operatorname{rand}(0,1] \times\left(U_{h_{j}}-U_{l_{j}}\right),\left(i=1,2 \ldots N P ; j=1,2 \ldots N_{k}\right)
$$

where $U_{i, G}$ is each individual in $G$ th generation, $U_{l_{j}}$ and $U_{h_{j}}$ are the lower and upper bounds of control variables in dimension $j . N P=200$ is the size 
of population, whereas $N_{k}=50$ is the number of time nodes. In order to select the next population $U_{i, G+1}$, the target vector $U_{j, G}$ competes with the corresponding trial vector $U_{i, G}$ by using the modified game theory, which will be further discussed in Section 3.2.

Following the population initialization, the mutation strategy is applied 175 to generate a mutant vector $v_{i, G}$ with respect to the target vector $U_{i, G}$. The mutation operator can be described as:

$$
v_{i, G}=\gamma U_{i, G}^{*}+(1-\gamma) U_{i, G}+F \sum_{i=1}^{K}\left(U_{i_{a}^{k}}-U_{i_{b}^{k}}\right)
$$

where $\gamma \in[0,1]$ is the greediness of the individual. $U_{i, G}^{*}$ is the best individual in the $G$ th generation and $K$ is the number of differentials applied to calculate the perturbation. The control parameter $\gamma$ stands for the degree of exploitation and greediness of the mutation operator. That is, if $\gamma$ value is large, it is expected to emphasize the exploitation ability of the algorithm and vice versa. Another control parameter $F$ controls the diversity and exploration ability of the mutation process, which means the larger $F$ value is, the higher the diversity of exploration can be. According to some recent work [32, $F$ should be chosen in a tight region between 0.4 and 1 . Taking this into consideration, the range of $F$ value in the proposed method is set in $[0.4,1.0]$.

As for the crossover strategy, there are two types of crossover methods employed in differential evolution (DE) community: binomial and exponential crossovers. From existing literature [33, it is found that the exponential crossover tends to be more sensitive with respect to the problem size than the binomial method. Since the size of discrete optimal control problem depends largely on the number of time nodes, the binomial crossover is conducted in the proposed method.

$$
U_{i j, G}^{\prime}= \begin{cases}v_{i j, G}, & \text { if } \operatorname{rand}(0,1)<C R \\ U_{i j, G}, & \text { otherwise. }\end{cases}
$$

where $C R$ is the crossover rate and is set $C R=0.3$. The quality of a multiobjective evolutionary algorithm consists of three design goals [23, 34]. Since most of the multi-objective algorithms are based on nondominated solutions, the distance of the resulting nondominated set to the true pareto-optimal front should be minimized. Moreover, a good distribution of the obtained solutions found is desirable. Last, the extent of the obtained nondominated front should 
the length of diagonal hyperbox formed by the extreme solution observed in the current pareto front. The maximum speed can be set as:

$$
V_{\text {max }}=\left(\frac{1}{M} \sum_{m=1}^{M}\left(\frac{\max _{i=1}^{Q} f_{m}^{i}-\min _{i=1}^{Q} f_{m}^{i}}{F_{m}^{\max }-F_{m}^{\min }}\right)^{2}\right)^{\frac{1}{2}}
$$

where $M$ is the number of objective function, whereas $Q$ the observed pareto set. Correspondingly, $f_{m}^{i}$ is the value of the $m$ th objective function of the $i$ th number 225 of $Q . F_{m}^{\max }$ and $F_{m}^{\min }$ are the maximum and minimum values of the current 
optimal solution, respectively. Since all the quality factors of a multi-objective algorithm are converted to mathematical expressions, the next step is to design an adaptive control logic in order to enhance the convergence ability and quality of the solution. The input value is the change of performance metrics generated every two successive generation, whereas the output is the trends of control parameters $F$ and $\gamma$. For simplicity reasons, the relationships are described as "increase", "decrease" and "no change". This adaptive control logic can be summarised in Table 1. The control logic illustrated in Table 1 is used for

Table 1: Adaptive control logic

\begin{tabular}{c|c|c|c|c|c}
\hline \multirow{2}{*}{ No.logic } & \multicolumn{3}{|c|}{ Input } & \multicolumn{2}{c}{ Output } \\
\cline { 2 - 6 } & $N F_{\text {known }}$ & $\mathrm{S}$ & $V_{\max }$ & $\mathrm{F}$ & $\gamma$ \\
\hline 1 & Increase & Increase & Increase & No change & Increase \\
2 & Increase & Decrease & Increase & No change & No change \\
3 & Increase & Increase & Decrease & No change & Decrease \\
4 & Increase & Decrease & Decrease & Increase & No change \\
5 & Decrease & Increase & Increase & No change & Decrease \\
6 & Decrease & Decrease & Increase & Increase & No change \\
7 & Decrease & Increase & Decrease & Increase & Decrease \\
8 & Decrease & Decrease & Decrease & Increase & No change \\
\hline
\end{tabular}

adjusting the control parameter $F$ and $\gamma$ in order to emphasize the greediness or diversity of the mutation strategy. Specifically, based on the performance metrics, if it is desired to emphasize the exploitation, the value of $\gamma$ should be increased and $F$ should be decreased. On the other hand, if it is desired to enhance exploration, $\gamma$ is decreased while $F$ is increased. The logic "No change" can be used to $\gamma$ or $F$ when it is desired to have a mild emphasis in terms of exploitation or exploration. It should be noted that logic number 2 is the best case because hypervolume is increasing and spacing is decreasing, which implies that the population is converging. Therefore, the control parameters $\gamma$ and $F$ remain unchanged. On the other hand, logic number 7 is the worst case scenario since all the quality factors indicate that the current front is diverging and losing diversity and extensiveness. Therefore, it is desired to emphasize exploration so to decrease $\gamma$ and increase $F$. 


\subsection{Modified game theory}

The selection process is conducted in a competitive way. A modified game theory is applied to make a comparison between different individuals and conthe game theory, namely non-cooperative and cooperative game theory. Each objective function is associated with a player. Thus, the $i$ th player aims to optimize his/her own objective function $J_{i}$. In this paper, it is assumed that all the players agree to find a compromised solution according to a mutually agreeable 
sup-criterion can be expressed as:

$$
S=\prod_{k=1}^{M}\left(1-\mu_{J_{i}}(U)\right)
$$

Hereafter, a new fitness function can be created to find a Pareto optimal solution, which can represent a compromised solution.

$$
F=\Phi-S
$$

\begin{tabular}{|c|c|c|c|c|c|}
\hline & $J_{1}$ & $J_{2}$ & $J_{3}$ & $\cdots$ & $J_{m}$ \\
\hline Opt $J_{1}(x, u)$ & $J_{1}\left(x_{1}^{*}, u_{1}^{*}\right)$ & $J_{2}\left(x_{1}^{*}, u_{1}^{*}\right)$ & $J_{3}\left(x_{1}^{*}, u_{1}^{*}\right)$ & $\cdots$ & $J_{m}\left(x_{1}^{*}, u_{1}^{*}\right)$ \\
\hline Opt $J_{2}(x, u)$ & $J_{1}\left(x_{2}^{*}, u_{2}^{*}\right)$ & $J_{2}\left(x_{2}^{*}, u_{2}^{*}\right)$ & $J_{3}\left(x_{2}^{*}, u_{2}^{*}\right)$ & $\cdots$ & $J_{m}\left(x_{2}^{*}, u_{2}^{*}\right)$ \\
\hline Opt $J_{3}(x, u)$ & $J_{1}\left(x_{3}^{*}, u_{3}^{*}\right)$ & $J_{2}\left(x_{3}^{*}, u_{3}^{*}\right)$ & $J_{3}\left(x_{3}^{*}, u_{3}^{*}\right)$ & $\cdots$ & $J_{m}\left(x_{3}^{*}, u_{3}^{*}\right)$ \\
\hline & $\vdots$ & $\vdots$ & $\vdots$ & $\vdots$ & . \\
\hline Opt $J_{m}(x, u)$ & $J_{1}\left(x_{m}^{*}, u_{m}^{*}\right)$ & $J_{2}\left(x_{m}^{*}, u_{m}^{*}\right)$ & $J_{3}\left(x_{m}^{*}, u_{m}^{*}\right)$ & $\cdots$ & $J_{m}\left(x_{m}^{*}, u_{m}^{*}\right)$ \\
\hline
\end{tabular}

Table 2: Payoff table

Using the adaptive differential evolution algorithm based on modified game theory, the pareto optimal set can be calculated. The flow chart of the proposed method is presented in Fig 3.

\section{Simulation results}

The initial state boundary conditions of the skip process are set as $x_{0}=$ $\left[r_{0}, \theta_{0}, \phi_{0}, V_{0}, \gamma_{0}, \psi_{0}, m_{0}\right]=[6450451.9 \mathrm{~m}, 0 \mathrm{deg}, 0 \mathrm{deg}, 7802.9 \mathrm{~m} / \mathrm{s},-1 \mathrm{deg}, 90 \mathrm{deg}$, $92078.8 \mathrm{~kg}$. The final altitude point is set as $r_{f}=21162900 \mathrm{ft}$. Box constraints for each state variable are set as $x_{\min }=[21066900 \mathrm{ft},-180 \mathrm{deg},-70 \mathrm{deg}, 609.6 \mathrm{~m} / \mathrm{s}$, $-80 \mathrm{deg},-180 \mathrm{deg}, 20000 \mathrm{~kg}]$ and $x_{\max }=[6450451.9 \mathrm{~m}, 180 \mathrm{deg}, 70 \mathrm{deg}, 13716 \mathrm{~m} / \mathrm{s}$, $80 \mathrm{deg}, 180 \mathrm{deg}, 92078.8 \mathrm{~kg}$ ], respectively. It is worth noting that the same parameter setting can be found in [20]. The specific boundary conditions were set specifically at the start of the project for this type of missions. In order to verify the feasibility of proposed method, only the first hop is taken into account in the paper. The initial altitude is around $80 \mathrm{~km}$ where is the assumed edge of atmosphere.

Firstly, to generate the payoff table for the modified game theory, the optimization results for each single objective function are generated using the adaptive differential evolution algorithm. The results are shown in Fig.4 to 7. 


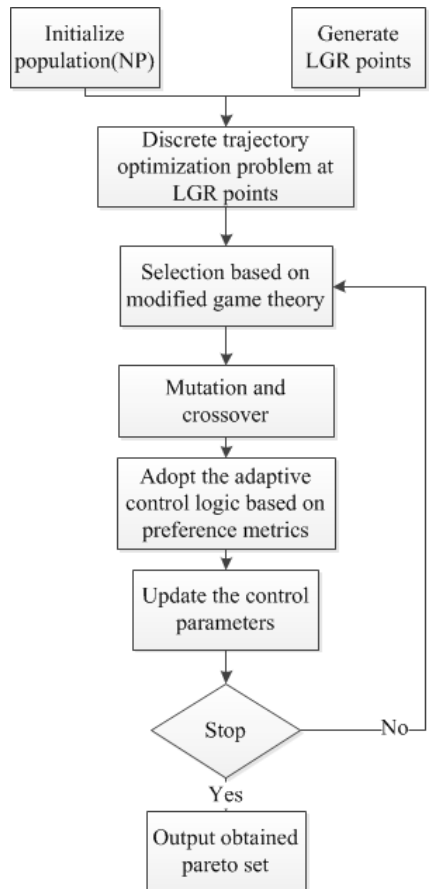

Figure 3: Flow chart of the proposed algorithm

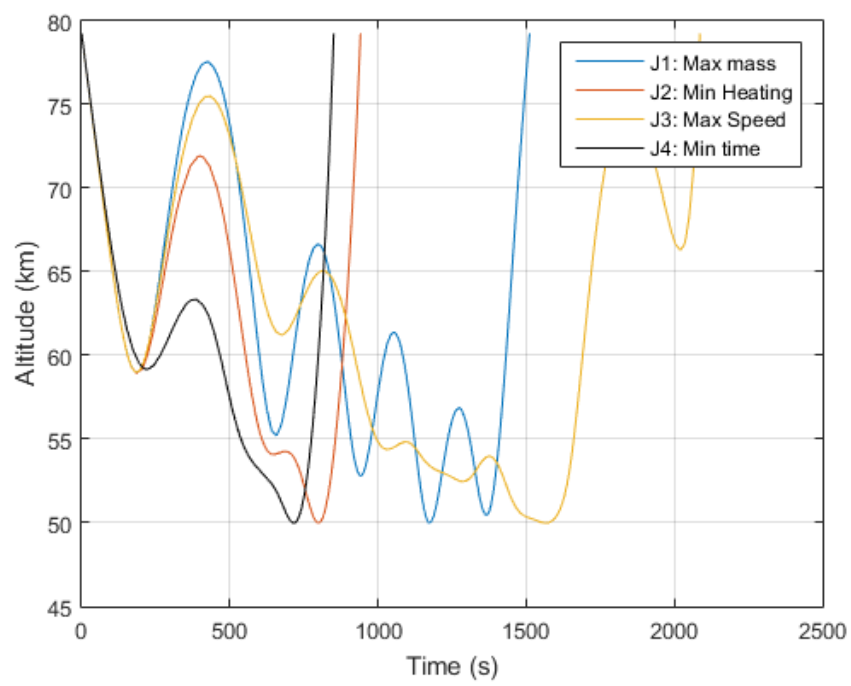

Figure 4: Altitude for different objective function 


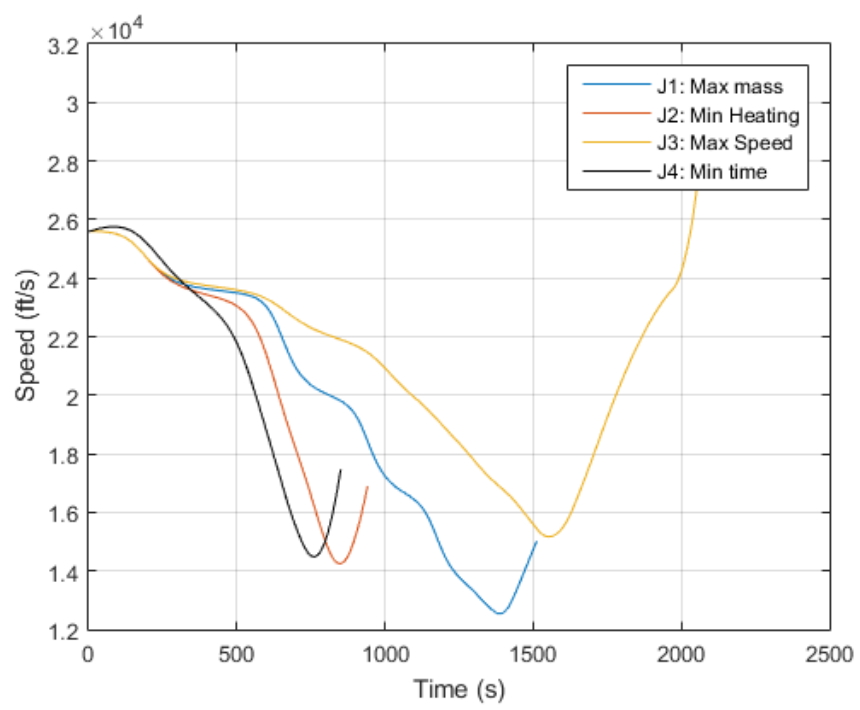

Figure 5: Speed for different objective function

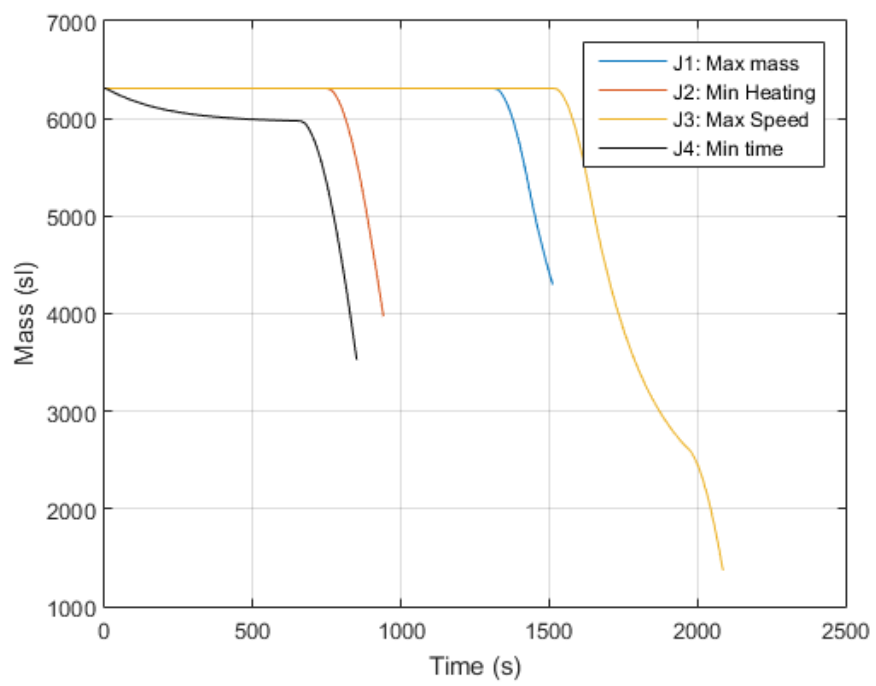

Figure 6: Mass for different objective function

As can be seen from Fig. 4 to 7, conflicts exist between each objective function, therefore it is impossible to find a solution optimizing each cost function. More precisely, in the case of maximizing final velocity, if there is no compro- 


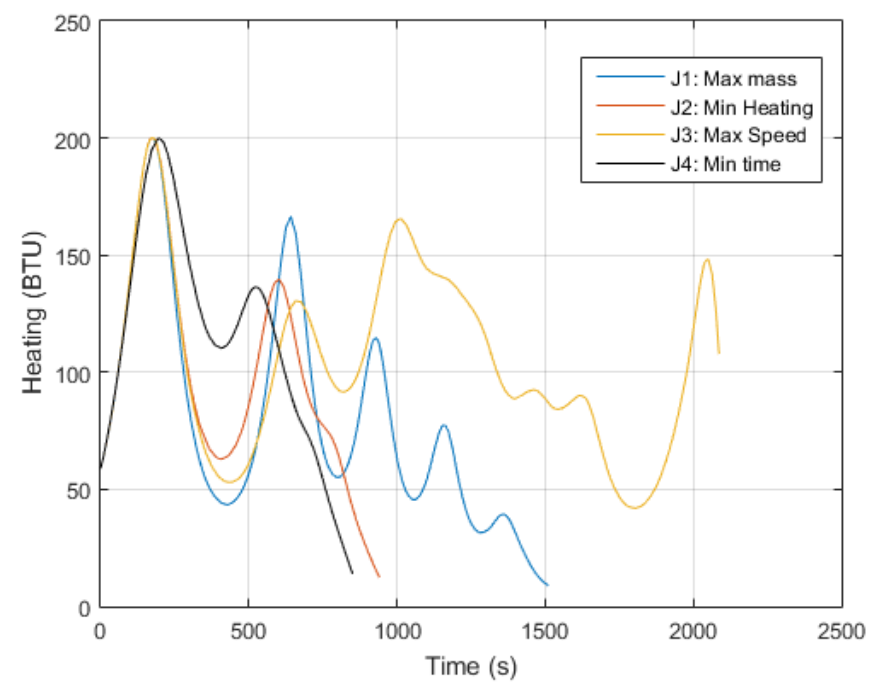

Figure 7: Heating for different objective function

mised procedure, then to achieve higher speeds, the SMV is tending to complete the mission taking longer time and accelerating during the whole time period. That implies it is trying to consume all the fuel (see e.g. Fig.6). Hence, after reaching the low earth orbit, the SMV has no fuel left to continue the mission. Based on the single-objective solution, the following payoff table is constructed (see e.g. Table 3).

Table 3: Payoff table

\begin{tabular}{c|c|c|c|c}
\hline \hline & $J_{1}$ & $J_{2}$ & $J_{3}$ & $J_{4}$ \\
\hline $\min J_{1}$ & 851.65 & 89.23 & 17862.4 & 3563.2 \\
\hline $\min J_{2}$ & 942.84 & 75.65 & 16978.6 & 4042.8 \\
\hline $\max J_{3}$ & 2085.41 & 219.32 & 30846.8 & 1370.4 \\
\hline $\max J_{4}$ & 1502.03 & 123.86 & 15012.8 & 4297.5 \\
\hline
\end{tabular}

\subsection{Pareto front}

From the payoff table [20], the worst value $F_{i}$ and optimum solution $J_{i}^{*}$ can be obtained: $F_{1}=2085.41, F_{2}=219.32, F_{3}=15012.8$ and $F_{4}=1370.4$, whereas $J_{1}^{*}=851.65, J_{2}^{*}=75.65, J_{3}^{*}=30846.8$ and $J_{4}^{*}=4297.5$, respectively. 
Using these solutions, the modified game theory model can be constructed. The optimized solution of multi-objective SMV trajectory optimization problem are

(5) ADEMGT (proposed): Adaptive differential evolution based on modified game theory.

It is worth noting that in 20, the NSGA2 method was applied to generate the pareto front of the multi-objective SMV trajectory optimization problem. studies. During the optimization procedure, the pareto front is generated as the set of solutions that has the highest fitness value and is not dominated by any other solutions in the current population. By limiting the computational effort for all of the algorithms (computational time), the Pareto fronts, generated by MOEA/D-DE, NSGA2, SPEA2, NPGA and proposed ADEMGT algorithms, are projected onto two planes as shown in Fig.8 and Fig.9.

By checking the constraint profiles, all the methods stated above manage to generate skip entry trajectories between the predetermined initial position and terminal position without violating the path constraints. Therefore, the structure integrity of the SMV can be guaranteed and all the solutions calculated by applying different multi-objective evolutionary algorithms (ADEMGT) can be accepted as feasible solutions. As can be seen from Fig.8 and Fig.9, the adaptive differential evolution based on modified game theory method generally performs better than other multi-objective evolutionary algorithms for solving the SMV 


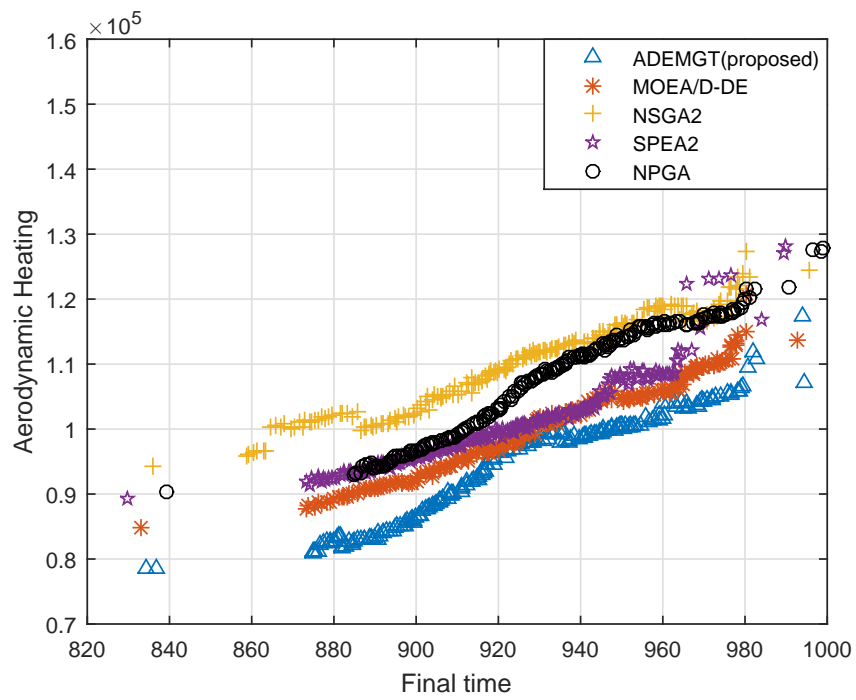

Figure 8: Pareto front generated using six algorithms

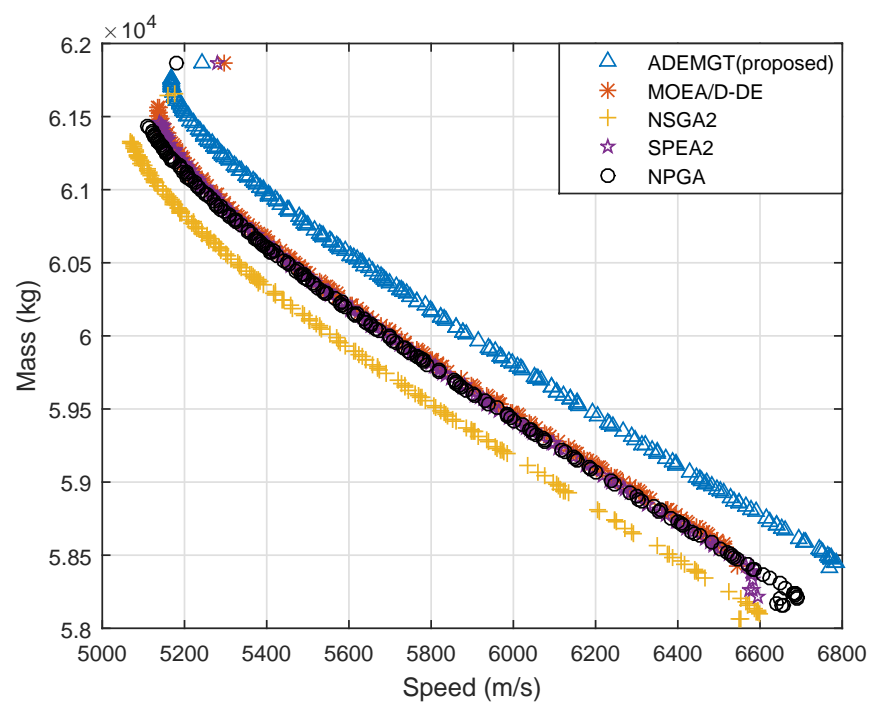

Figure 9: Pareto front generated using six algorithms

trajectory optimization problem. This can be reflected by the quality of generated pareto front. Specifically, the pareto set calculated by applying ADEMGT can cover the pareto front calculated using other evolutionary algorithms. Based 
on the control logic described in Section 3, the control parameters, $F$ and $\gamma$, are updated during the iteration process, which means the exploitation and exploration of the pareto front can be emphasized adaptively. Hence, the quality of multi-objective solution can be guaranteed by implementing the proposed method.

\subsection{Convergence ability}

Another important factor to qualify the algorithm is the convergence ability. The history of the fitness value was projected onto the fitness versus iteration time plane shown in Fig.10. Generally, the fitness value of all the

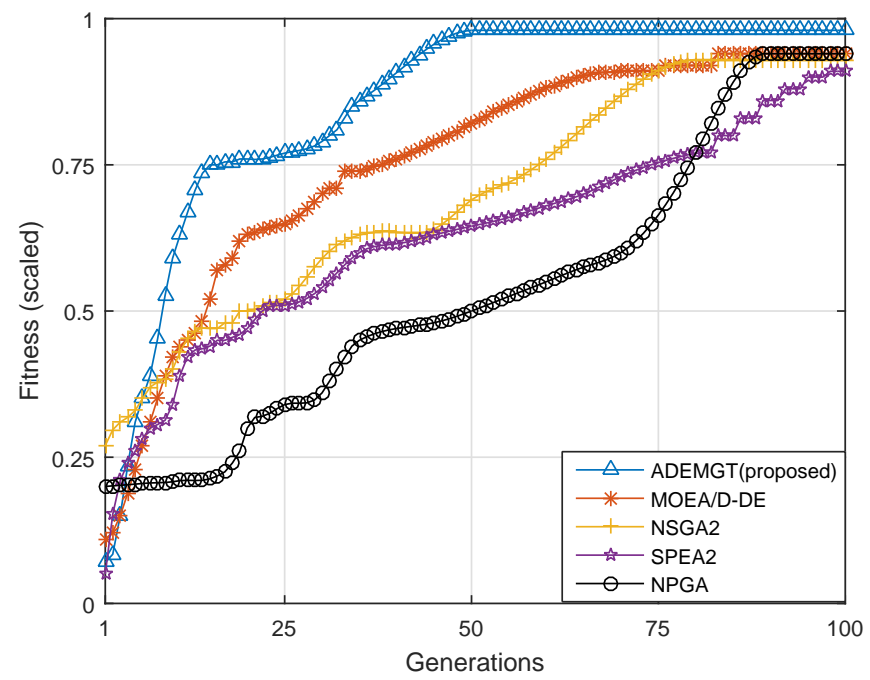

Figure 10: Fitness versus iteration time

multi-objective evolutionary algorithms tend to converge to around 1, which means all the stated methods can converge in finite iterations. From Fig.10, the final value of fitness function of the proposed ADEMGT method is higher than its counterparts. Moveover, since by applying the modified game theory, it can not only simplifies the computational procedure using a super-criterion, but also considers the solution of the general single-objective optimization problems involving all the design variables. Therefore, compared with other methods tested in this paper, the ADEMGT can always achieve a quicker convergence speed (less generations to converge). That implies by using limited computa- 
tional efforts, the quality of solutions generated from ADEMGT is better than other methods. This is important especially when the computational burden must be taken into account in practical uses.

\subsection{Algorithm stability}

365

In order to analyze the algorithm stability, a series of simulations are conducted by testing three different target position scenarios. More specifically, the target radii are set as $r_{1}=21066900 \mathrm{ft}(6421.2 \mathrm{~km}), r_{2}=21074900 \mathrm{ft}(6423.6 \mathrm{~km})$ and $r_{3}=21080900 \mathrm{ft}(6425.5 \mathrm{~km})$ for each scenario. Fig.11 to 13 illustrate the convergence results of the proposed method for each scenario in terms of fitness function versus iteration time pane.

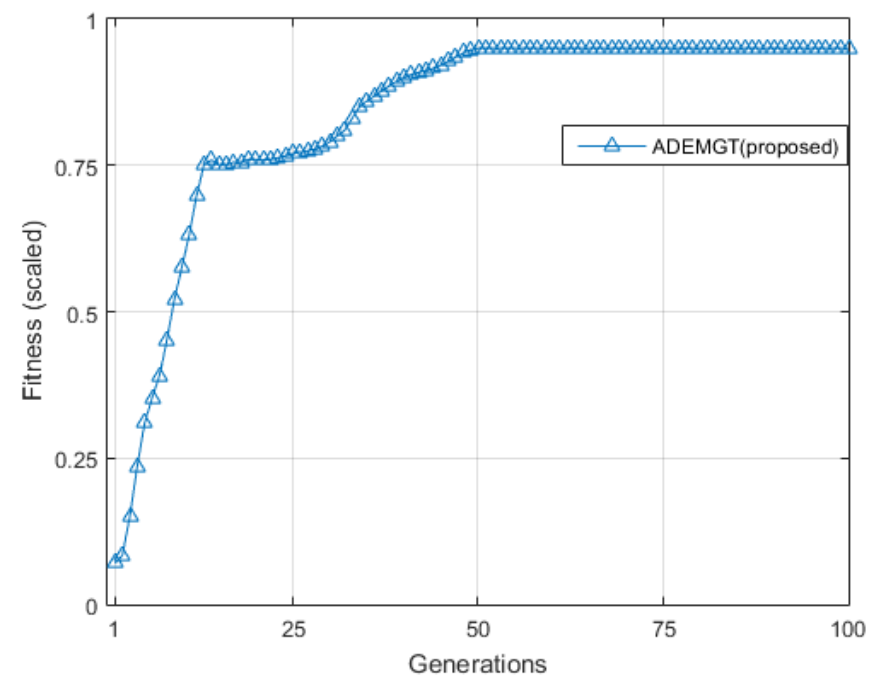

Figure 11: Fitness versus iteration time (Scenario 1: Radius $=6421.2 \mathrm{~km}$ )

It can be seen from Fig.11 to 13 that the proposed algorithm tend to converge after finite generations for each scenario. These results confirm that the ADEMGT algorithm is not sensitive with respect to different boundary conditions and can be used to generate compromised solution for multi-objective trajectory optimization problems.

In summary, all the figures and data provided earlier confirm the feasibility of the proposed adaptive differential evolution based on modified game theory 


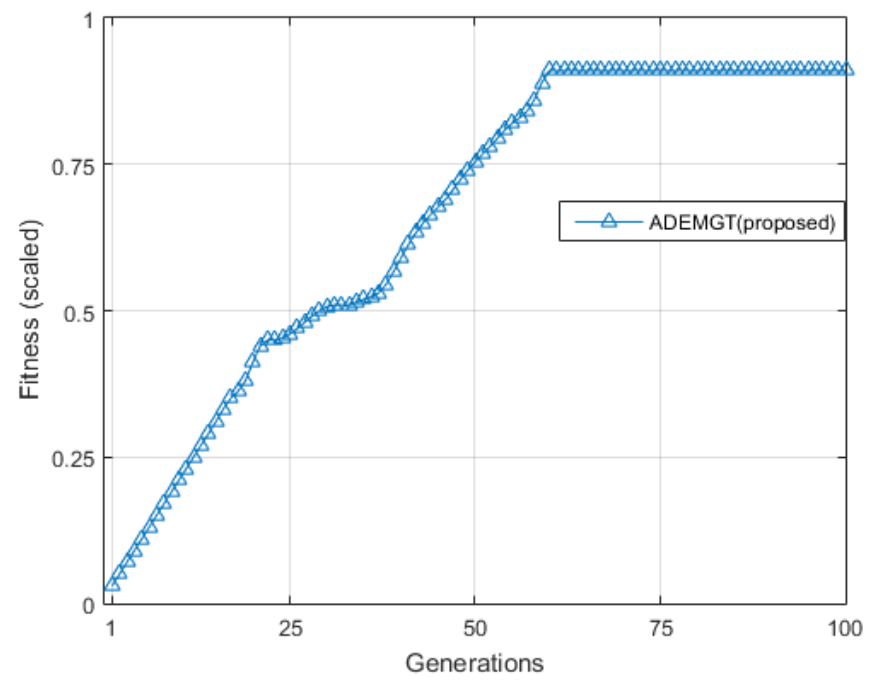

Figure 12: Fitness versus iteration time (Scenario 2: Radius $=6423.6 \mathrm{~km}$ )

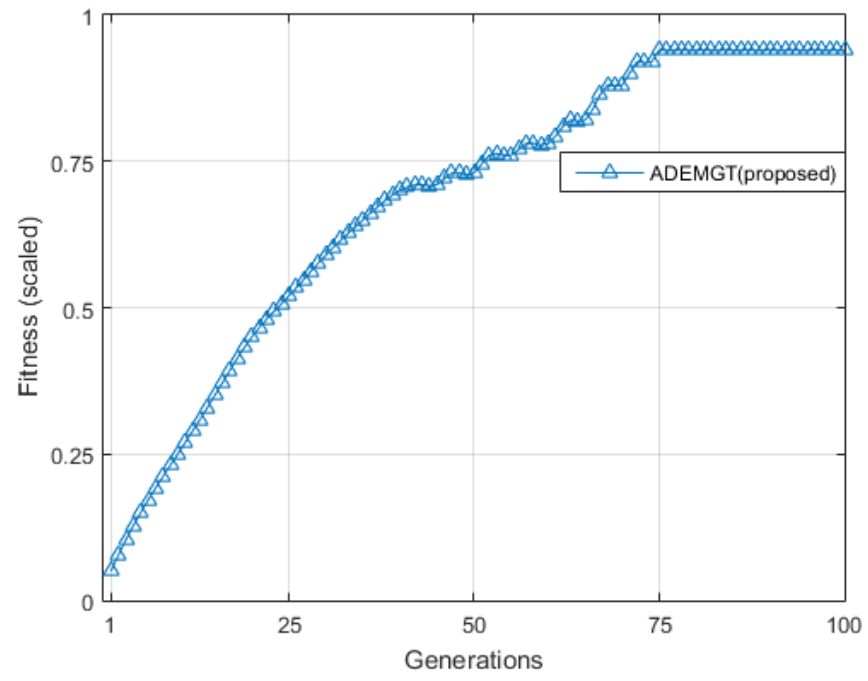

Figure 13: Fitness versus iteration time (Scenario 3: Radius $=6425.5 \mathrm{~km}$ )

method. By using different multi-objective evolutionary optimization strategies, the SMV can reach the target position and return back to the low Earth 380 orbit without violating path constraints and boundary conditions. In addition, 
it is shown that the proposed algorithm has better convergence ability and convergence speed than other strategies tested in this paper, which means the compromised trajectories calculated using ADEGMT are feasible and reliable.

\section{Conclusions}

385

In this paper, the original single-objective trajectory optimization model was extended to multi-objective optimization formulation, including minimum final time, aerodynamic heating, maximum final velocity and mass, in order to design a near optimal trajectory, which can capture multiple requirements for SMV. In order to transcribe the continuous-time optimal control problem, a specific multiple shooting method is applied to discrete the dynamics. An adaptive differential evolution based on modified game theory algorithm is then designed to solve the resulting nonlinear programming problem. This algorithm uses an adaptive strategy to update the mutation operator and applies a modified game theory to guide the evolution procedure. In addition, to control the algorith$\mathrm{m}$ and improve the quality of the solution, a control logic is embedded in the proposed method framework. Simulation results show that compared with other multi-objective evolutionary approaches, the proposed algorithm has better performance in terms of generating high quality pareto front. Also, by applying the control logic and modified game theory, the proposed algorithm can have a better convergence ability and quicker convergence speed than other approaches without violating any path and box constraints. Then, the proposed method is efficient and effective to solve the SMV trajectory optimization problem.

\section{References}

[1] C. W. Brunner, P. Lu, Skip entry trajectory planning and guidance, Journal of Guidance, Control, and Dynamics 31 (5) (2008) 1210-1219. doi:10. $2514 / 1.35055$

[2] L. Bruyere, A. Tsourdos, B. A. White, Polynomial approach for design and robust analysis of lateral missile control, International Journal of Systems Science 37 (8) (2006) 585-597. doi:10.1080/00207720600784742.

[3] L. Guarnaccia, R. Bevilacqua, S. P. Pastorelli, Suboptimal lqr-based spacecraft full motion control: Theory and experimentation, Acta Astronauti- 
ca 122 (2016) 114-136. doi:http://dx.doi.org/10.1016/j.actaastro. 2016.01 .016 .

[4] P. Lu, Predictor-corrector entry guidance for low-lifting vehicles, Journal of Guidance, Control, and Dynamics 31 (4) (2008) 1067-1075. doi:10.2514/ 1.32055

[5] B. Tian, Q. Zong, Optimal guidance for reentry vehicles based on indirect legendre pseudospectral method, Acta Astronautica 68 (7) (2011) 11761184. doi:http://dx.doi.org/10.1016/j.actaastro.2010.10.010.

[6] A. Tsourdos, R. Zbikowski, B. A. White, Robust autopilot for a quasilinear parameter-varying missile model, Journal of Guidance, Control, and Dynamics 24 (2) (2001) 287-295. doi:10.2514/2.4711.

[7] P. Lu, Entry guidance using time-scale separation in gliding dynamicn s, Journal of Spacecraft and Rockets 52 (4) (2015) 1253-1258. doi: $425 \quad 10.2514 / 1 . A 33295$.

[8] P. Shi, Limit hamilton-jacobi-isaacs equations for singularly perturbed zerosum dynamic (discrete time) games, SIAM Journal on Control and Optimization 41 (3) (2002) 826-850. doi:10.1137/s036301290037908x.

[9] J. T. Betts, Survey of numerical methods for trajectory optimization, Jour${ }_{430}^{4} \quad$ nal of Guidance, Control, and Dynamics 21 (2) (1998) 193-207. doi: $10.2514 / 2.4231$.

[10] J. T. Betts, W. P. Huffman, Mesh refinement in direct transcription methods for optimal control, Optimal Control Applications and Methods 19 (1) (1998) 1-21.

${ }_{435}$ [11] C. L. Darby, W. W. Hager, A. V. Rao, Direct trajectory optimization using a variable low-order adaptive pseudospectral method, Journal of Spacecraft and Rockets 48 (3) (2011) 433-445. doi:10.2514/1.52136.

[12] D. Garg, M. A. Patterson, C. Francolin, C. L. Darby, G. T. Huntington, W. W. Hager, A. V. Rao, Direct trajectory optimization and costate estimation of finite-horizon and infinite-horizon optimal control problems using a radau pseudospectral method, Computational Optimization and Applications 49 (2) (2009) 335-358. doi:10.1007/s10589-009-9291-0. 
[13] J. Laurent-Varin, F. Bonnans, N. Berend, M. Haddou, C. Talbot, Interiorpoint approach to trajectory optimization, Journal of Guidance, Control, and Dynamics 30 (5) (2007) 1228-1238. doi:10.2514/1.18196.

[14] Y. Zhao, P. Tsiotras, Density functions for mesh refinement in numerical optimal control, Journal of Guidance, Control, and Dynamics 34 (1) (2011) 271-277. doi:10.2514/1.45852.

[15] P. Williams, Jacobi pseudospectral method for solving optimal control problems, Journal of Guidance, Control, and Dynamics 27 (2) (2004) 293297. doi:10.2514/1.4063

[16] D. A. Benson, G. T. Huntington, T. P. Thorvaldsen, A. V. Rao, Direct trajectory optimization and costate estimation via an orthogonal collocation method, Journal of Guidance, Control, and Dynamics 29 (6) (2006) 1435-1440. doi:10.2514/1.20478.

[17] R. Jeremy, Launch Vehicle Trajectory Optimization Using a Legendre Pseudospectral Method, Guidance, Navigation, and Control and Co-located Conferences, American Institute of Aeronautics and Astronautics, 2003, doi:10.2514/6.2003-5640. doi:doi:10.2514/6.2003-5640.

[18] Q. Gong, F. Fahroo, I. M. Ross, Spectral algorithm for pseudospectral methods in optimal control, Journal of Guidance, Control, and Dynamics 31 (3) (2008) 460-471. doi:10.2514/1.32908.

[19] M. A. Patterson, W. W. Hager, A. V. Rao, A ph mesh refinement method for optimal control, Optimal Control Applications and Methods 36 (4) (2015) 398-421. doi:10.1002/oca.2114.

[20] R. Chai, A. Savvaris, A. Tsourdos, Fuzzy physical programming for space manoeuvre vehicles trajectory optimization based on hp-adaptive

(1) pseudospectral method, Acta Astronautica 123 (2016) 62-70. doi:http: //dx.doi.org/10.1016/j.actaastro.2016.02.020

[21] H. Li, Q. Zhang, Multiobjective optimization problems with complicated pareto sets, moea/d and nsga-ii, IEEE Transactions on Evolutionary Computation 13 (2) (2009) 284-302. doi:10.1109/TEVC.2008.925798. 
[22] A. Tsourdos, E. J. Hughes, B. A. White, Fuzzy multi-objective design for a lateral missile autopilot, Control Engineering Practice 14 (5) (2006) 547561. doi:http://dx.doi.org/10.1016/j.conengprac.2005.01.009.

[23] K. Deb, A. Pratap, S. Agarwal, T. Meyarivan, A fast and elitist multiobjective genetic algorithm: Nsga-ii, IEEE Transactions on Evolutionary Computation 6 (2) (2002) 182-197. doi:10.1109/4235.996017.

[24] A. Elhossini, S. Areibi, R. Dony, Strength pareto particle swarm optimization and hybrid ea-pso for multi-objective optimization, Evolutionary Computation 18 (1) (2010) 127-156. doi:10.1162/evco.2010.18.1.18105.

[25] N. Padhye, P. Mittal, K. Deb, Feasibility preserving constraint-handling strategies for real parameter evolutionary optimization, Computational Optimization and Applications 62 (3) (2015) 851-890. doi:10.1007/ s10589-015-9752-6.

[26] C. Jariyatantiwait, G. G. Yen, Fuzzy multiobjective differential evolution using performance metrics feedback, in: 2014 IEEE Congress on Evolution-

n ary Computation (CEC), 2014, pp. 1959-1966. doi:10.1109/CEC. 2014. 6900533

[27] J. Wang, H. Peng, P. Shi, An optimal image watermarking approach based on a multi-objective genetic algorithm, Information Sciences 181 (24) (2011) 5501-5514. doi:http://dx.doi.org/10.1016/j.ins.2011.07.040.

[28] H. Peng, J. Wang, Z. Zhang, P. Shi, Audio watermarking framework using multi-objective particle swarm optimization, International Journal of Innovative Computing Information and Control 9 (7) (2013) 2789-2800.

[29] C. Gang, X. Min, W. Zi-ming, C. Si-lu, RLV Reentry Trajectory MultiObjective Optimization Design Based on NSGA2 Algorithm, Guidance, Navigation, and Control and Co-located Conferences, American Institute of Aeronautics and Astronautics, 2005. doi:10.2514/6.2005-6131.

500 [30] N. Yokoyama, S. Suzuki, Modified genetic algorithm for constrained trajectory optimization, Journal of Guidance, Control, and Dynamics 28 (1) (2005) 139-144. doi:10.2514/1.3042. 
[31] H. Gao, X. Yang, P. Shi, Multi-objective robust h-infinity control of spacecraft rendezvous, IEEE Transactions on Control Systems Technology 17 (4) (2009) 794-802. doi:10.1109/TCST.2008.2012166.

[32] Y. Wang, Z. Cai, Q. Zhang, Differential evolution with composite trial vector generation strategies and control parameters, IEEE Transactions on Evolutionary Computation 15 (1) (2011) 55-66. doi:10.1109/TEVC.2010. 2087271.

[33] D. Zaharie, Influence of crossover on the behavior of differential evolution 【 algorithms, Applied Soft Computing 9 (3) (2009) 1126-1138. doi:http: //dx.doi.org/10.1016/j.asoc.2009.02.012.

[34] M. T. Jensen, Reducing the run-time complexity of multiobjective eas: The nsga-ii and other algorithms, IEEE Transactions on Evolutionary Computation 7 (5) (2003) 503-515. doi:10.1109/TEVC.2003.817234.

[35] E. Zitzler, M. Laumanns, L. Thiele, Spea2: Improving the strength pareto evolutionary algorithm.

[36] J. Horn, N. Nafpliotis, D. E. Goldberg, A niched pareto genetic algorithm for multiobjective optimization, in: Evolutionary Computation, 1994. IEEE World Congress on Computational Intelligence., Proceedings of the First IEEE Conference on, 1994, pp. 82-87 vol.1. doi:10.1109/ICEC. 1994.350037

[37] M. A. Abido, A niched pareto genetic algorithm for multiobjective environmental/economic dispatch, International Journal of Electrical Power \&

525 \\Energy Systems 25 (2) (2003) 97-105. doi:http://dx.doi.org/10.1016/ S0142-0615(02) 00027-3. 
2017-03-24

\section{Multi-objective trajectory optimization of Space Maneuver Vehicle using adaptive differential evolution and modified game theory}

Chai, Runqi

Elsevier

Chai R, Savvaris A, Tsourdos A, Chai S, Multi-objective trajectory optimization of Space Maneuver Vehicle using adaptive differential evolution and modified game theory, Acta pÿAstronautica, Volume 136, July 2017, Pages 273280.

http://dx.doi.org/10.1016/j.actaastro.2017.02.023

Downloaded from Cranfield Library Services E-Repository 